\title{
Evaluation of new streptococcal latex grouping kit
}

\author{
A F Vicca, R E Stansfield, R G Masterton
}

\begin{abstract}
Aims: To evaluate a new streptococcal latex grouping kit (Shield Diagnostics Ltd) and compare it against an established latex agglutination method (Streptex; Wellcome Diagnostics).

Methods: Two hundred and forty seven strains of streptococci and enterococci were tested with each kit by one operator and according to the manufacturer's instructions. Strains failing to group or giving discordant results were identified to species level.
\end{abstract}

Results: Two discrepant grouping results were observed and 13 non- $\beta$ haemolytic streptococci failed to group with either product. The Shield kit successfully identified 232 isolates at 15 minutes of enzyme extraction incubation compared with 224 and 233 on short (15 minutes) and long (1 hour) incubations, respectively, for Streptex ( $p>0.23$ for both comparisons). On short incubation only, the Shield kit detected significantly more strains of Enterococcus faecium $(p=0 \cdot 007)$. The reaction strengths were similar for both kits $(p>0 \cdot 16)$. No cross-reactions were observed but the Streptex kit produced significantly fewer tests with visible granularity $(p<0.003)$.

Conclusions: Although the Shield product appeared to detect group $D$ antigen more readily, overall no important differences in performance were observed. Prospective users of the new method should first become familiar with its characteristics.

(f Clin Pathol 1993;46:79-80)

Since Lancefield first demonstrated the value of streptococcal serological grouping, ${ }^{1}$ faster and simpler methods have been sought. ${ }^{23}$ Pronase-B extraction ${ }^{45}$ and latex agglutination methods ${ }^{67}$ have made streptococcal grouping feasible for most laboratories. This report compares a new latex agglutination kit, the Shield Diagnostics Streptococcal Identification kit (SDSI), (Shield Diagnostics Ltd., Dundee) with the Streptex latex agglutination kit (WS) (Wellcome Diagnostics, Dartford).

Microbiological

Laboratories, Western

General Hospital,

Crewe Road,

Edinburgh, EH4 2XU

A F Vicca

R E Stansfield

R G Masterton

Correspondence to:

Dr R G Masterton

Accepted for publication 13 July 1992
Two hundred and eleven consecutive streptococcal clinical isolates and 12 strains of Enterococcus faecium with 24 strains of group G streptococci taken from stored clinical isolates were tested. Organisms were tested directly from the primary plates or, failing this, after overnight subculture on Columbia horse blood agar at $35^{\circ} \mathrm{C}$ in air with $5 \% \mathrm{CO}_{2}$. All isolates were tested, by a single operator (AFV), using the manufacturer's recommendations.

A light suspension of the organism was prepared in $400 \mu \mathrm{l}$ extraction enzyme solution (McFarland 0.5 to 1 for SDSI and growth equivalent to at least 5 large colonies for WS). This was incubated for 15 minutes at $37^{\circ} \mathrm{C}$ and shaken after 5 minutes. The latex reagents were shaken and one drop of each of the six groups dispensed onto a separate well of a clean reaction card. Thereafter, one drop of extraction solution (about $20 \mu \mathrm{l}$ ) was added to each well, the two were mixed, and spread to cover the entire reaction area. Finally, the card was gently rocked for 1 minute and examined, by naked eye, for visible agglutination. Any isolate failing to group by the WS kit was retested after an hour's extraction incubation according to the manufacturer's instructions. Any isolate failing to group with either or both kits, or giving discrepant results, was formally identified by API 20 Strep (BioMèrieux, Basingstoke).

Reaction strengths were recorded as strong, weak, trace and no reaction. Results were analysed by Yates' corrected $\chi^{2}$ tests and the Fisher exact test where appropriate.

\section{Results}

The overall results of grouping by each kit are shown in table 1 -combined long ( 1 hour) and short (15 minutes) incubation in the case of WS compared with SDSI (15 minutes) incubation. Two discrepancies were observed: on 1 hour incubation WS identified a strain of $E$ faecium (trace group D reaction) and another isolate, subsequently identified as Streptococcus mitis, as a trace group F on short incubation, but both failed to group with SDSI.

Thirteen non- $\beta$ haemolytic streptococci failed to group with either kit. These were identified as $S$ milleri $(\mathrm{n}=5), S$ mitis $(\mathrm{n}=3), S$ sanguis $(\mathrm{n}=3), S$ bovis and $S$ morbillorum (one each). In total, WS successfully identified 233 isolates but only 224 after short incubation, with one false positive result. SDSI successfully identified 232 isolates after short incubation but missed one strain of $E$ faecium. There was no significant difference between the number of strains grouped by SDSI and either the short or combined WS results $(p>0.23)$. Similarly, there were no statistical differences between the grouping results for the following organism subsets: (i) $\beta$ haemolytic streptococci 
Table 1 Overall grouping results ( $n=247$ isolates)

\begin{tabular}{|c|c|c|c|c|c|c|c|c|}
\hline \multirow{2}{*}{\multicolumn{2}{|c|}{ Streptococcal group }} & \multicolumn{7}{|c|}{ SDSI kit } \\
\hline & & $A$ & $B$ & $C$ & $D$ & $F$ & $G$ & $F T G^{\star}$ \\
\hline WS kit & $\begin{array}{l}\text { A } \\
\text { B } \\
\text { C } \\
\text { D } \\
\text { F } \\
\text { G } \\
\text { FTG }\end{array}$ & 50 & 49 & 24 & 48 & 10 & 51 & $\begin{array}{r}1 \\
1 \\
13\end{array}$ \\
\hline
\end{tabular}

^Failed to group

\section{Discussion}

The SDSI kit performed as well as the commercially available WS kit in terms of reaction strength, isolate numbers grouped, and result correlation. Although SDSI identified more organisms by short incubation than WS and produced more "strong" reactions, neither difference was significant.

SDSI appeared to detect group D antigen more readily-significantly more strains of Enterococcus faecium were identified after short incubation. This may also be reflected in the non-specific granularity seen most commonly in the D well, particularly with the SDSI kit, and $B$ isolates. Granularity did not complicate interpretation as its quality and slowness to develop differentiated it easily from true agglutination. However, the SDSI inoculum is critical and we experienced troublesome crossreaction agglutination if heavier than recommended suspensions were used.

The trial protocol did not include a compar-

Table 3 Occurrence of visible granularity by kit and streptococcal group

\begin{tabular}{lll}
\hline & \multicolumn{2}{l}{$\begin{array}{l}\text { Reaction showing granularity (No of } \\
\text { isolates) }\end{array}$} \\
\cline { 2 - 3 } Streptococcal group (No) & WS kit & SDSI kit \\
\hline A (50) & D (1) & - \\
B (49) & D (13) & D (34) \\
C (24) & D (2), A/F (1) & D (1), AD/F (1) \\
D (49) & G (2) & G (2) \\
F (10) & D (5) & D/G (1) \\
G (51) & D (9), C (1) \\
\hline
\end{tabular}

excluding $\beta$ haemolytic $S$ milleri $(\mathrm{n}=167)$; (ii) non- $\beta$ haemolytic strains excluding $E$ faecium and non- $\beta$ haemolytic $S$ milleri $(n=42)$; (iii) Streptococcus milleri strains $(n=17)$.

Evaluation of the short incubation reaction strengths, for all the above comparisons, showed no significant differences $(p>0 \cdot 16)$ (table 2).

The only such difference in kit performance, was found with $E$ faecium where, after short incubation, SDSI grouped $14 / 15$ as group D compared with $7 / 15$ by WS (Fisher exact test; $p=0.007)$. This difference, however, was not maintained after prolonged incubation, when WS correctly grouped 15/15 (Fisher's exact test; $\mathrm{p}=0.5$ ).

No cross-reactions were detected, but visible granularity was observed significantly more often with SDSI ( $p<0.003$ ). The single group where this reached significance was with $B$ reactions $(p<0.00006)$ (table 3$)$. ison of reaction strengths between kits but results with the SDSI kit were generally more pronounced at each grade. This observation does however, need, to be formally evaluated.

The test procedures were very similar and this, taken with the above, means there is little difference between the kits. However, the SDSI kit is shown to have its own characteristics so centres considering its use must not view it as a straight replacement for their current system but should first become familiar with its performance.

This project was supported by Shield Diagnostics Ltd.

1 Lancefield RC. A serological differentiation of human and other groups of haemolytic streptococci. $\mathcal{F}$ Exp Med 1933;57:571-95.

2 Fuller AT. The formamide method for the extraction of polysaccharides from haemolytic streptococci. $B r \mathcal{F} \operatorname{Exp}$ Pathol 1938;19:130-9.

3 El Kholy A, Wannamaker LW, Krause RM. Simplified extraction procedure for serological grouping of betahaemolytic streptococci. Appl Microbiol 1974;28:836-9.

4 Ederer GM, Herrmann MM, Bruce R, Matsen JM Chapman SS. Rapid extraction method with pronase B for grouping beta-haemolytic streptococci. Appl Microbiol 1972;23:285-8.

5 Watson BK, Moellering RC, Kunz LJ. Identification of streptococci: use of lysozyme and Streptomyces albu filtrate in the preparation of extracts for Lancefield grouping. $\mathcal{f}$ Clin Microbiol 1975;1:274-8.

6 Lue YA, Howitt IP, Ellner PD. Rapid grouping of betahaemolytic streptococci by latex agglutination. $\mathcal{f}$ Clin Microbiol 1978;8:326-8.

7 Facklam RR, Cooksey RC, Wortham EC. Evaluation of commercial latex agglutination reagents for grouping streptococci. $\mathcal{F}$ Clin Microbiol 1979;10:641-6. 